\title{
Acquired Hemophilia in Female - A Case Report
}

\author{
MADHABI KARMAKER, ${ }^{1}$ ISRAT ZERIN, ${ }^{2}$ SALMA AFROSE, ${ }^{3}$ MD. MUJIBUR RAHMAN, ${ }^{4}$ KHAN ABUL KALAM AZAD 5
}

\begin{abstract}
:
Acquired hemophilia is a rare but acquired bleeding diathesis caused by autoimmune depletion of Factor VIII or IX.Diagnosis of Acquired Hemophilia requires clinical acumen. The condition is often under diagnosed as because it is rare and patients usually don't have any unusual personal or family history of bleeding episodes.Acquired hemophilia has an equal sex distribution. So, clinicians should suspect acquired hemophilia in any patients with unexplained persistent and profound bleeding from uncommon soft tissues and mucosa and having prolonged activated partial thromboplastin time (APTT) without other causes. Here we will report a 50-year-old lady presenting with recurrent spontaneous bruises and joint pain and swelling for 2 years. On examination we found multiple bruises and ecchymosis of different sizes with varying colour changes from bluish to greenish over different parts of her body including face, right shoulder, right elbow joint and $2^{\text {nd }}$ and $3^{\text {rd }}$ PIP joints. Lab findings showed decreased Hemoglobin with prolonged APTT which didn't correct in mixing study.After exclusion of other possible pathological conditions and on the basis of lab criteria we diagnosed the case as Acquired Hemophilia. She was successfully treated with blood transfusion, recombinant activated factor VIII, Steroid and supportive treatments and was discharged with improving symptoms, stable hematocrit and resolving bruises.
\end{abstract}

Keywords: Acquired hemophilia, bleeding diathesis, activated partial thromboplastin time (APTT).

\section{Introduction}

Acquired Hemophilia (AH) is a rare condition in which auto antibodies, usually of the IgG class are produced against factor VIII or IX. Most antibodies bind to the 44DA2 domain and/or the 72 KDC2 domain of factor VIII which is associated with insufficient generation of thrombin through intrinsic pathway of coagulation cascade and increased bleeding diathesis. Acquired Hemophilia is significantly rarer than the inherited form, affecting around 2 per million of the population. It appears in all ethnic groups and has a worldwide prevalence. ${ }^{1,2,3,4,5}$ Age distribution is bimodal with a first peak occurring among young women in the post partum period and a second major peak among elderly patients. ${ }^{3}$ The mortality rate is high, ranging from $8 \%$ $22 \%$. This is related to severe hemorrhage that can occur in $85 \%-90 \%$ of the patients. ${ }^{3,4}$ There is often an underlying medical condition associated with this Acquired Hemophilia. An association with other autoimmune conditions (SLE, Inflammatory bowel disease, Rheumatiod arthritis), malignant disease, certain drugs (such as penicillin,

1. Indoor Medical officer, Dept. of Medicine (Unit 1), Dhaka Medical College \& Hospital (DMCH), Dhaka

2. Post graduate trainee, Dept. of Medicine, DMCH, Dhaka.

3. Professor of Hematology, DMCH, Dhaka.

4. Professor of Medicine, DMCH, Dhaka.

5. Professor and Head, Dept. of Medicine, DMCH, Dhaka.

Corresponding author: Dr. Madhabi Karmaker, Indoor Medical Officer, Dept of Medicine, Dhaka medical College hospital, Dhaka. Email:meru7010@yahoo.com. sulphonamide, interferon, fluderabine, phenytoin, flupentixol etc), pregnancy and Infections ( $\mathrm{HBV}, \mathrm{HCV}$ ) has been recognized in various surveys. In approximately half of all cases, there is no obvious underlying cause and the condition is labeled as idiopathic. ${ }^{3,6,7,8,9,10}$

The pattern of bleeding in $\mathrm{AH}$ also differs from that of inherited hemophilia. Bleeding tends to occur in soft tissue, muscle, retroperitoneal space and gastrointestinal or genitourinary tracts. In contrast patients with hereditary hemophilia, patients with hemarthrosis are rare. ${ }^{3,4}$ Bleeding after very minor trauma is also common. The reason for the completely different bleeding pattern in Acquired Hemophilia remains unknown and there is no demonstrable impairment of platelet function.

Given the complexity of diagnosis, the condition may be under diagnosed for many years. ${ }^{11,12,13,14}$ The typical laboratory findings of Acquired Hemophilia are a prolonged activated partial thromboplastin time, normal prothrombin time as well as normal platelet count and function. Mixing studies can be used to demonstrate the presence the of a time dependent inhibitor of factor VIII and IX and the antibody titers are measured by Bethesda test.

Treatment of Acquired Hemophilia is two -pronged. The immediate priority is to control acute bleeding with bypassing agents. ${ }^{4-11}$ Immunosuppressant should be used to control antibody production. Oral steroids $1 \mathrm{mg} / \mathrm{kg}$ for 3-6 weeks can cause remission in one third to one half of patients with Acquired hemophilia. ${ }^{3,15,16,17}$ Human factor VIII is likely 
to be very rapidly inactivated by a significant titer of inhibitory antibody and therefore of no practical use, even at high doses. It is reported that most deaths in Acquired Hemophilia occurs in the first few weeks. Thus a prompt recognition of the disorder and an early and aggressive treatment are mandatory.

\section{Case Report}

A 50 year old lady, housewife, hailing from Begumgonj, Noakhali district was admitted into Medicine department of Dhaka Medical College Hospital with the complaints of pain in different joints, and recurrent spontaneous bruises for 2 years. She first noticed sudden swelling of her left ankle associated with pain. Pain was dull aching with moderate severity, aggravated on movement and relieved by taking NSAIDS. Simultaneously her right ankle joint and both knees became swollen. The overlying skin of the joint was bluish in colour along with some spontaneous bruises in different parts of her both lower limbs. The conditions persisted for few days and gradually pain and swelling of joints disappeared with sequential colour changes of bruises over about 2 months. Similar type of episodes occurred at variable time intervals involving different parts of her body. Last episode occurred 1 day before admission when her whole face became enormously swollen with bluish black discoloration after a slap over her face by her husband. At that time she also presented with myalgia and painful swelling of right shoulder joint, right elbow joint, and $2^{\text {nd }}$ and $3 \mathrm{rd}$ PIP joints of right hand with overlying bruises without any precipitating illness or trauma. On further enquiry, she complained of gum bleeding while brushing her teeth very rarely over last 20 years, increased menstrual flow and duration of 8-9 days with regular cycle with normal frequency over 2 years with no previous menstrual abnormality. She also reported hair loss of about $50 \%$ over this period. As the disease progressed she gradually developed pallor, and generalized weakness. She had no bad obstetric history including spontaneous abortion. She has 2 sons, 3 daughters and 6 sisters and 3 brothers, none of the family members including maternal and paternal uncles have similar complaints. Her parents had no history of consanguinity of marriage.

On examination, she was ill looking, depressed with average body built. Her whole face was swollen, bluish black in colour, tender on palpation with black eye. She also had multiple bruises and ecchymosis of different sizes with varying colour changes from bluish to greenish over different parts of the body. She was moderately anemic, pulse-82 / min, BP- 100/72mmHg, Temperature- normal, RR-14 breaths/min, JVP- not raised, no accessible lymph nodes were palpable. Her musculoskeletal system examination revealed that right shoulder, right elbow joint and $2^{\text {nd }}$ and $3^{\text {rd }}$ PIP joints of right hand were swollen, tender with raised temperature and overlying skin was bluish in colour. Movements of the joints were restricted secondary to pain. After disappearance of joint swelling we again examined the patient and found no signs of sinovitis. All other systemic examinations revealed no abnormality.

According to investigation profile, complete blood count revealed - severe anemia (on $30.0614 \mathrm{Hb}: 5.8 \mathrm{mg} / \mathrm{dl}$, Hct $18.1 \%$ ) which was gradually corrected after 5 units of blood transfusion (Hb: $11.7 \mathrm{~g} / \mathrm{dl}$ and Hct $35.6 \%$ on 17.07 .14 ; Hb: $11.1 \mathrm{mg} / \mathrm{dl}$, Het $34.8 \%$ on 10.08 .14$)$; neutrophilic leukocytosis but normal platelet count.

WBC on 30.06.2014- 12,520/ul-N: $65 \%$, L: $26 \%$

17.07.2014 - 19, 550/ul- N 85\%, L: $9 \%$

10.08.2014-6, 830/ul-N 45\%, L : $43 \%$.

Platelet count on 30.06.2014- 3,79,000/ul.

Peripheral blood film showed normocytic anaemia with neutrophilic leukocytosis with increased rouleaux formation. Coagulation profile after admission (on 3.7.2014) showed prolonged APTT (80 sec) with normal BT (3 min $30 \mathrm{sec})$, CT $(2$ mins $20 \mathrm{sec})$, TT (16 sec) and PT( $17 \mathrm{sec})$. Other examination revealed-SGPT $22 \mathrm{U} / 1$, Serum creatinine 2.85 $\mathrm{mg} / \mathrm{dl}$ (on 01.07.2014)

$\mathrm{O} .84 \mathrm{mg} / \mathrm{dl}$ (on 23.08.14)

Urine R/ M/ E: Normal, ANA \& Anti Ds DNA

- Negative, OGTT - Normal

USG of Whole abdomen revealed Cholelithiasis, bilateral echogenic renal parenchyma and multiloculated cyst in left ovary.

Further coagulation profile revealed -

APTT $76.2 \mathrm{sec}$ (Normal : $28 \mathrm{sec)} 70 \mathrm{sec} 65 \mathrm{sec}$

Factor VIII activity $2.7 \%(\mathrm{~N}: 50-150 \mathrm{sec}) 1 \%$

Factor IX activity 2\% (N : 50- $150 \%$ ) $10.3 \%$

At the same time we assessed Von Willebrand factor level which was found to be normal.

VWF : 114\% ( N: 50- 160\%)

Then we went for Mixing Assay which revealed the following

\section{Test Result Test result 2 hours after incubation}

APTT 1:1 dilution $66.8 \mathrm{sec} 70.6 \mathrm{sec}$

Factor VIII 1:1 dilution 33\% 2.6\%

Factor IX 1: 1 dilution $59 \% 249 \%$

Interpretation of mixing Study...

Immediate \% Incubated\% Suggests

Correction Correction

$>50 \%>10 \%$ Factor deficiency

$<50 \%>10 \%$ Mild Factor deficiency 
$>50 \%<10 \%$ factor Inhibitor

$<50 \%<10 \%$ Lupus anticoagulant

As interpretation of this mixing study was highly suggestive of presence of lupus anticoagulant, we measured the serum level and it was found to be moderately present.

Lupus anticoagulant $1.6(<1.2=$ absent $)$

1.2-1.5 weakly present

1.5-2.0= moderately present

$>2.0=$ Strongly present

\section{Discussion}

The incidence of Acquired Hemophilia increases with age with a peak incidence in $7^{\text {th }}$ and $8^{\text {th }}$ decades of life though there is a small peak in the $3^{\text {rd }}$ decade likely corresponding to post partum inhibitors. Initially in this case, we had some differentials considering the history and clinical exam, like-

1. Hemorrhagic disorder with possible systemic Lupus erythematosus.

2. Von willebrand disease and

3. Acquired coagulation disorder.

Along with bruises and ecchymosis a female patient with initial presentation of joint pain and swelling, hair loss of about $50 \%$ over the whole period, gradual pallor and weakness made us to think about SLE with hemorrhagic disorder, but absence of rash, photosensitivity, oral ulceration, no bad obstetric history, normal urine R/M/E findings and negative ANA and Anti-DS DNA virtually excluded the diagnosis of SLE.

Then after doing the coagulation profile and finding a prolonged APTT with normal BT, CT, PT and TT, we considered two additional differentials-

Von willebrand disease and Acquired coagulation disorder.

Then we assessed Von willebrand factor level as well as factor VIII and IX activity level. A normal VWF level excluded Von willebrand disease.As Factor VIII and IX were found to be deficient there were two possibilities either inherited deficiency of factor VIII and IX or acquired deficiency due to presence of inhibitors. A female patient with negative family history with bleeding episodes at this age decreased the possibility of Inherited Hemophilia. We decided to go for Mixing study. Mixing study is usually done to differentiate inherited factor deficiency from presence of inhibitors. While performing mixing study we should be careful of the nonspecific inhibitor Lupus Anticoagulant which causes multiple factor deficiency.
A prolonged APTT which initially corrects in a 1: 1 mix but again prolonged after 2 hours incubation -is suggestive of inhibitors against factor VIII and IX but a prolonged APTT which fails to be corrected in 1:1 mix initially and also after incubation is suggestive of presence of lupus anticoagulant,which is the most frequently encountered inhibitor.

In our case, as the Mixing study failed to correct prolonged APTT in a 1:1 mix immediately and also after 2 hours incubation, Lupus anticoagulant was the most likely cause. Then we measure Lupus anticoagulant level and it was found to be moderately present. At this point we needed Bethesda test to quantify the inhibitors but unavailability of this facility made us unable to do the test. Though LA was present but the patient had no features of thrombosis, no bad obstetric history and had negative Antiphospholipid antibody, so antiphospholipid antibody syndrome was unlikely. We also didn't find any underlying autoimmune disease or other medical conditions or drugs behind this illness. Thus we leveled our case as Idiopathic Acquired Hemophilia.

Treatment should be focused in Acquired Hemophilia on controlling the immediate bleeding episode and suppressing the immune reaction against coagulation factor. The principal products available for the treatment of bleeding episodes are activated prothrombin complex concentrate (PCC), Factor VIII inhibitor bypassing agent (FEIBA) or recombinant activated factor VII. Recombinant factor VIIa interacts with thrombin activated platelets to produce a thrombin burst leading to accelerated fibrin clot formation localized to the site of vascular injury. The dose should be determined by the severity of the bleeding. Here it should be noted that even extensive cutaneous purpura doesn't necessarily require treatment. For suppressing of inhibitors recommended immunosuppressive agent is prednisolon $(1 \mathrm{mg} / \mathrm{kg})$ as soon as the diagnosis is established. Alternative options are Azathioprine, Rituximab, Cyclophosphamide, Mycophenolate, Cyclosporin etc. ${ }^{17-22 ; 23-26}$

Considering all the possibilities we treated our patient after admission with tranexamic acid, inj dexamethasone and inj ceftriaxone along with other supportive measures. We also provided oral Vit $\mathrm{C}$ considering possible vit $\mathrm{C}$ deficiency as well as vascular fragility in older age. We transfused about 5 units of whole blood to stabilize $\mathrm{Hb}$ level and Hct concentration. Then we also transfused 2 units of factor VIII concentrates and we started steroid at the dose of $1 \mathrm{mg} / \mathrm{kg}$ BW for 6 weeks. With all these treatments our patient gradually improved which was proved clinically and by the laboratory profile. 


\section{Conclusion}

Any patient with recurrent bleeding episodes with unexplained prolonged aPTT, Acquired hemophilia should be suspected and a limited panel blood tests should be conducted to identify any underlying condition. Acquired Hemophilia is a rare disorder and the potential for significant bleeding problem is high. It is therefore recommended that such patients should be managed in specialized hemophilia units, which have the necessary expertise and blood products available. It also needs to be remembered that many of the patients with this condition are elderly and frail and may thus be partially vulnerable to the adverse effects of treatment with steroids.

\section{Conflict of interest: None.}

\section{References}

1. Delgado J, Yimenez-YusteV, Hernandez-NavarroF, Villar A. Acquired hemophilia: review and metaanalysis focused on therapy and prognostic factors. Br J Haematol 2003;121:2135 .

2. Collins P, Macartney N, Davies B, Lees S, Giddings J, Maier R. A population based, unselected, consecutive cohort of patients with acquired hemophilia A. BrJ Hematol 2004;124:86-90.

3. Massimo Franchini and Giuseppe Lippi. How I treat Acquired factor VIII inhibitors, blood 2008;112: 250-255.

4. Green D, Lechner K. A survey of 125 nonhemophilic patients with inhibitors to factor VIII. Thromb haemost 1981;45:200203.

5. Sallah S, Nguien NP, Abdullah JM, hanrahan I.R. Acquired hemophilian patients with hematologic malignancies.Arch pathol Lab Med 2000;124:730-4.

6. Massimo franchini, Giovanni Targher, Franco manzato. Acquired factor VIII inhibitors in oncohematology: A systemic review. Crit Rev Owcol hematol 2008;66:194-199.

7. Sallah S, Wan JY. Inhibitors against factor VIII in patients with cancer. Cancer 2001;91: 1067-74.

8. Meiklejohn DJ, Watson HG, Acquired hemophilia in association with organ specific autoimmune disease. Hemophilia 2001;7:523-5.

9. Hultin MB. Acquired inhibitors in malignant and nonmalignant disease states. Am J Med 1991;91:9-13.

10. Peter W, Collins, Sybil Hirsch, Trevor P, Baglin. Acquired haemophilia $\mathrm{A}$ in the united Kingdom: a 2 year national surveillance study by the United kingdom hemophilia centre Doctors' Organisation. Blpood 2007;109:1870-1877.
11. Paul Giangrande. Acquired haemophilia. Published by the World Federation of hemophilia (WFH) 2005.

12. Giuseppe Tagariello \& Roberto Sartori \& Paolo Radossi. Intenssive blood transfusion support in acquired hemophilia A. Ann Hematol 2007;86:229-230.

13. Sallah S. Treatment of acquired hemophilia with factor eight inhibitor bypassing activity. Hemophilia 2004;10:169-173.

14. P.A. Holme, F.Brosstad, G.E Tjonnfjord. Acquired hemophilia : management of bleeds and immune therapy to eradicate autoantibodies. Hemophilia 2005;11:510-515.

15. Brian Barnett, Rebecca Kruse-Jarres and cindy A. Leissinger. Current management of acquired factor VIII inhibitors. Curr Opin Hematol 2008;15:451-461.

16. Spero JA, Lewis JH, Hasiba U, Corticosteroid therapy for acquired F VIII: C inhibitors. Br J Hematol 1981;48:635-42.

17. Stasi R, Bruneti M, Stipa E, Amadori S, Selective B-cell depletion with Rituximab for the treatment of patients with acquired hemophilia. Blood 2004;103:4424-4428.

18. Abdallah A, CoghIan DW, Duncan EM, Chunilal SD, et al. Rituximab induced long term remission in patients with refractory acquired hemophilia. J Thromb Haemost 2005;3:2589-2590.

19. Wiestner A, Cho HJ, Asch AS et al. Rituximab in the treatment of Acquired factor VIII inhibitors. Blood 2002;100:3426-3428.

20. Garvey B, Rituximab in the treatment of autoimmune hematological disorders. Br J Hmetol 2008;141:149-169.

21. Franchini M, Rituximab in the treatment of adult acquired hemophilia A: a systematic review. Crit Rev Oncol Hematol 2007;63:47-52.

22. Aggarwal A, Grewal R, Green R, et al. Rituximab for autoimmune hemophilia: a proposed treatment algorithm. Haemophilia 2005;11:13-9.

23. Schulman S, Langevitz P, Livneh U, Mortinowitz U, Seligsohn U, Varon D, Cyclosporine therapy for acquired factor VIII in hibitor in apatient with Systemic Lupus Erythematosus. Thromb Haemost 1996;76:344-6.

24. Pfiegler g, Boda Z, Harsfalvi J, et al. Cyclosporin therapy of a women with acquired hemophilia due to factor VIII: C inhibitor. Postgrad Med J 1989;65:400.

25. Petrovic M, Derom E, Baele G. Cyclosporine treatment of acquired hemophilia due to factor VIII antibodies. Haematologica 2000;85:895-896.

26. Au WY, Lam CC, K wong YL. Successful treatment of acquired factor VIII inhibitor with cyclosporine. Hemophilia. 2004;10:98-100. 